\title{
Evaluation of non-invasive arterial stiffness parameters and their relationship with physical activity, anthropometric indices and lipid profiles in healthy middle-aged adults: results of the PERSIAN cohort study
}

Mohammad Nosrati-Oskouie ${ }^{1}$, Sajjad Arefinia ${ }^{2}$, Saeed Eslami Hasan Abadi $^{2}$, Abdolreza Norouzy $^{2}$, Hamed Khedmatgozar ${ }^{3}$, Nazanin Sadat Aghili-Moghaddam², Maryam Alinezhad-Namaghi ${ }^{2}$, Mohammad Reza Shadmand Foumani Moghadam ${ }^{4}$, and Reza Rezvani $^{2}$

${ }^{1}$ Tabriz University of Medical Sciences

${ }^{2}$ Mashhad University of Medical Sciences

${ }^{3}$ Texas Tech University

${ }^{4}$ Varastegan Institute for Medical Sciences

January 2, 2021

\begin{abstract}
Background: Arterial stiffness (AS) indicates the initial stage of cardiovascular disease (CVD), which associated with modifiable and lifestyle risk factors. We aimed to examine the association of AS with anthropometric indices, lipid profiles, and physical activity. Methods: 658 healthy middle-aged adults selected and anthropometric indices (body mass index (BMI), waist circumferences (WC), waist-to-hip ratio (WHR), waist-to-height ratio (WHtR), neck circumferences (NC), a body shape index (ABSI), body roundness index (BRI), body-fat mass (BFM), visceral-fat, fat-free mass(FFM), lipid profiles, and PA were measured. Arterial Stiffness measured by carotid-femoral pulse wave velocity (cf-PWV) and central augmentation index (cAIx). Results: Our results show, cf-PWV positively associated with TGs $(\beta=0.10, \mathrm{p}=0.01)$ and in anthropometric indices corelated with, WC $(\beta=0.11, p=0.02)$, WHR $(\beta=0.09, p=0.03)$, WHtR $(\beta=0.1, p=0.02)$, and BRI $(\beta=0.09, p=0.04)$. cAIx was independently positive association with cholesterol $(\beta=0.08, \mathrm{p}=0.03)$, WC $(\beta=0.1, \mathrm{p}=0.03)$, WHR $(\beta=0.09$, p =0.02), ABSI $(\beta=0.09, p=0.01)$, BRI $(\beta=0.08, p=0.05)$, visceral-fat area $(\beta=0.09, p=0.03)$ and BFM $(\beta=0.08, p=0.04)$ and negatively associated with PA $(\beta=-0.08, \mathrm{p}=0.03)$. Conclusions: WC, WHR, and BRI were associated with both cf-PWV and cAIx. TGs and WHtR associated with cf-PWV, while cAIx was associated with ABSI, so improving these indices may be helpful to prevent CVD.
\end{abstract}

\section{Introduction:}

Arterial stiffness (AS) is an early-stage marker of vascular aging (1), which is associated with changes in physical characteristics of the wall's artery, such as distensibility, complacency, and elasticity (2). It has been shown that AS is related to the underlying cause of cardiovascular diseases (CVD) such as chronic low-grade inflammation and endothelial dysfunction $(3,4)$. AS obtained noninvasively through measurements of the pulse wave velocity (PWV), which is considered the gold standard, and it has independent predictive value for all-cause and CVD mortality independent of previous CVD, age, and type 2 diabetes mellitus (5, 6).

The relationship between AS and modifiable lifestyle factors such as physical activity (PA) (7), anthropometric indices (8), and lipid profiles (9) is not clear. Previous studies have failed to establish the relationship 
between AS with PA and lipid profiles. Some studies reported a negative correlation between AS and PA (10), and some studies have shown no association between these two (11). On the relationship between lipid profiles and AS, Zhao et al. showed that a reverse association between only high-density lipoprotein cholesterol (HDL-C) and AS (9). In contrast, results from cross-sectional studies showed a direct association between AS and triglycerides (TGs) (12). There are suggestive findings from studies showed that the degree of obesity, assessed by body mass index (BMI) and waist circumference (WC), is a positive correlation with AS. In contrast, other studies failed to demonstrate any association between AS with BMI and WC $(8,12)$.

Mentioned studies evaluated the relationship between only one of the AS markers with one of the modifiable lifestyle risk factors (anthropometric measures, lipid profiles, or PA). So it seems necessary to conduct a comprehensive study to determine all of these risk factors. The purpose of the current study was to assess the relationship between both local and systemic AS markers, including cf-PWV and cAIx, with anthropometric indices, lipid profiles, and PA among healthy middle-aged adults.

\section{Materials and methods:}

\section{Study population}

We conducted a nested cross-sectional study within PERSIAN (Prospective Epidemiological Research Studies in Iran) cohort study. The original objectives of the cohort were to assess and improve the health of Mashhad University of Medical Sciences Staff. For the current study, 658 healthy Iranian adult participants (321 men and 337 women) from the PERSIAN Organizational Cohort study in Mashhad University of Medical Sciences. This population-based prospective organization cohort study was conducted among 10000 randomly selected participants aged 30-70 years in Mashhad, Iran (2017-2020) as part of the "Persian cohort" research project. Additional details about the PERSIAN Cohort Study explained elsewhere (13). Exclusion criteria were: self-report of previous cardiovascular disease and those with the presence of diabetes, history of severe diseases (All types of cancer, Estrogen Replacement Therapy, drug abuse or major psychiatric illness), using regular supplementation with any of the vitamins or minerals, pregnancy and lactating, clinical conditions that could hamper hemodynamic measurements (absence of femoral pulse, systolic BP $<90 \mathrm{mmHg}$ ) and other factors that may have influenced AS like extreme beliefs or behavior related to diet. The Ethics Committee of the Mashhad University of Medical Sciences approved this study (Code of Ethics: IR.MUMS.MEDICAL.REC.1397.322). All participants signed a consent form approved by the ethics committee.

\section{Assessment of demographic variables}

Validated questionnaires used to collect data for age, sex, smoking habits, use of hookah, alcohol consumption, and physical activity, as used for PERSIAN cohort study.

Assessment of dietary intake and Physical Activity

Using the 24-hour recalls : participants asked to remember all of the foods and drinks they have consumed on the previous day. The values listed for each meal are converted to grams and consumed grams as well as energy intake calculated based on Home-Scale Guide, for each individual. PA determined by using a validated Physical Activity Questionnaire consists of 28 items designed by Persian Cohort. According to the questionnaire instructions, the metabolic equivalent (MET) calculated based on units per hour per day. We classified participants into two groups based on the mean MET rates of participants (38.6 METs/hour/day), less than 38.6 METs/hour/day defined as low activity levels.

Assessment of under- and over-reporting of energy intake

Using the ratio of energy intake to resting metabolic rate (RMR) assessed under- and over-reporting of energy intake. RMR measured by indirect calorimetry (MetaLyzer 3B, Cortex, Leipzig, Germany). To assess the validity of the reported energy intake, we used the ratio of the energy intake to RMR. We used the values defined in the Black et al. and Goldberg et al. $(14,15)$ study to investigate the relative degree of underand over-reporting. Thus, the range of 1.35-2.39 identified as the normal report energy intake in this study. 
Measurement of anthropometric parameters and blood pressure

All the anthropometric measurements were taken while the participants are wearing only light clothes. Height measured without shoes to the nearest $0.1 \mathrm{~cm}$. Weight, BMI, and body composition were examined using bio-impedance analysis (InBody770, Biospance Co., Ltd. Seoul, Korea). Waist Circumference (WC) at the umbilicus in a standing position was measured directly on the skin to the nearest $0.1 \mathrm{~cm}$ with a flexible tape, without any pressure to the body surface. Waist-to-hip ratio (WHR) calculated by dividing WC by the hip circumference. Waist-to-height ratio $(\mathrm{WHtR})$ calculated as the WC $(\mathrm{cm})$ divided by the height $(\mathrm{cm})$. Neck circumference (NC) was measured using a flexible tape at the lower margin of the thyroid cartilage with head erect and eyes facing forward. A body shape index (ABSI) and body roundness index (BRI) was calculated using the following formulas $(16,17)$ :

$\mathrm{ABSI}=\frac{\mathrm{WC}}{\left(\mathrm{BMI}^{\frac{2}{3}} \times h \mathrm{eight}^{\frac{1}{2}}\right)}$
$\mathrm{BRI}=364.2365 .5 \times \sqrt{1-\frac{\left(\frac{\mathrm{WC}}{2 \pi}\right)^{2}}{(0.5 \times h \text { eight })^{2}}}$

After 15 minutes rest, blood pressure was measured and recorded twice using a standard sphygmomanometer.

Biochemical Assessments

Blood samples collected from all participants in potassium-EDTA vacuum tubes after 10-12 hours of fasting. Total cholesterol, high-density lipoprotein cholesterol, triglycerides, and fasting plasma glucose were determined using standard laboratory procedures and analyzed by using the standard analyzer (BT 1500; Biotecnica Instruments, Rome, Italy).

Arterial stiffness measurement

AS was evaluated, according to the guidelines, using the gold standard carotid-femoral pulse wave velocity by the Sphygmocor (SphygmoCor Cardiovascular Management System software version 1.3; AtCor Medical Pty. Ltd., Sydney Australia). Participants were studied in the morning in the supine position after resting for 5-10 minutes in a quiet and temperature-controlled room. According to the instructions of the device, SphygmoCor XCEL PWA uses a standard brachial cuff to measure brachial systolic and diastolic pressures, and capture a brachial waveform. The brachial waveform is then analyzed by SphygmoCor to provide a central aortic waveform. Central BP measurements such as central aortic systolic BP, central pulse pressure, and an augmentation index were also reported. To conduct a carotid-femoral PWV measurement, a cuff placed around the femoral artery of the patient to capture the femoral waveform, and a tonometer was used to capture the carotid waveform. The distance in millimeters from the patient's carotid artery to their sternal notch (Carotid to Sternal Notch) and also the distance from their sternal notch to the top edge of the femoral cuff (Sternal Notch to Cuff) is measured, and the velocity automatically determined by dividing the distance by the pulse transit time. After 10 seconds of simultaneous valid carotid tonometer signal, The SphygmoCor software derives the ascending aortic pressure wave, substantially equivalent to the pressure wave measured by an invasive catheter. From the aortic pressure wave, the number of valuable cardiovascular measurements is determined, including central aortic systolic pressure, aortic augmentation index and central pulse pressure. The PWV was calculated using the distance and mean time difference between the two recorded points.

The central augmented pressure calculated as the difference between the maximum systolic peak on the aortic pulse wave and the inflection point. The latter is defined as the merging point for the incident and reflected wave. The cAIx indicates as the augmented pressure divided by pulse pressure $(\mathrm{PP}) \times 100$.

\section{Statistical analysis}

All statistical analyses were conducted by using IBM SPSS statistical software, version 25.0. The normality of variables tested using the Kolmogorov-Smirnov criterion. Continuous variables with normal distribution were expressed as mean \pm standard deviation (SD), whereas skewed distribution was expressed as median 
(interquartile range), and Categorical variables were represented by frequency and percentage. Because plasma TGs were skewed, log transformation was used. Tests of a trend for continuous and categorical variables across s of each AS markers (as a median value in each tertile) were conducted using the linear regression and $\chi^{2}$ analyses, respectively.

We used the Pearson correlation test to check the correlation between AS markers with anthropometry, lipid profile, and PA. Linear regression was used to determine the relationship between AS markers with anthropometry, lipid profile, and physical activity. The adjusted model was defined for controlling the confounders including age, sex, systolic and diastolic BP, heart rate (HR), fasting plasma glucose (FPG), blood urea nitrogen (BUN), Creatinine, alanine aminotransferase (ALT), aspartate aminotransferase (AST), alkaline phosphatase (ALP), skeletal muscle mass, sleep duration, total energy, alcohol consumption, and smoking. The significance level for all tests was considered less than 0.05 .

\section{Results:}

\section{Baseline characteristics}

General characteristics of the participants among tertiles of AS markers presented in Table 1-2. Participants in the highest tertile of cf-PWV were older, more male. They had higher BMI, waist and neck circumferences, ABSI, BRI, fat-free mass, SBP, DBP, TGs, cholesterol, non-HDL-C, and non-HDL/HDL ratio compared to the lowest tertile. Participants in the third tertile of AS marker were older, had higher BRI, NC, visceral fat, BFM, FFM, cholesterol, HEL-C, and most of them were women, compared to those in the first tertile.

Comparing AS indices in different tertiles, we showed that there are no significant differences between males and females regarding their AS in tertiles (Fig 1).

Associations between anthropometric indices and AS markers

Table 3 shows the correlation among the anthropometric indices and AS markers. Univariate analysis showed that the cf-PWV was positively associated with BMI, WHR, WHtR, ABSI, BRI, waist and neck circumferences, and fat-free mass. cAIx is positively correlated with WHtR, BRI, visceral fat area, and BFM, fat-free mass, and negatively correlated with neck circumferences.

In the regression model, after adjustment for confounders, cf-PWV was positively associated with waist circumferences, WHR, BRI, and WHtR, as shown in Table 4. Also, there was a positive association between cAIx and waist circumferences, WHR, and ABSI. Besides, cAIx was positively associated with the visceral fat area and BFM.

Associations between lipid profiles and AS markers

Cf-PWV was positively associated with both TGs and cholesterol, and cAIx was only positively associated with HDL-C and cholesterol, but not TGs. Non-HDL-C was positively associated with both cf-PWV and cAIx, while the ratio of non-HDL-C/HDL-C was just positively associated with cf-PWV (Table 3). After adjustment for confounders, cf-PWV and cAIx positively associated with TGs and cholesterol, respectively. Regression analysis for HDL-C, non-HDL-C, and non-HDL-C/HDL-C ratio showed no significant relation with cf-PWV and cAIx (Table 4).

Associations between PA and AS markers

No correlations were observed between AS markers and PA, as shown in Table 3. While, Multivariable linear regression analysis demonstrated a significant negative association of cAIx, but not cf-PWV, with PA after adjustment for confounders (Table 4 ).

\section{Discussions:}

The present study assessed the association of both local and systemic AS, represented by cf-PWV and cAIx, respectively, with anthropometric indices, lipid profiles, and PA. Our data revealed a positive association between AS markers and some parameters of the anthropometric index and lipid profiles. 
Lipid profile is associate with the Arterial Stiffness indexes:

Dyslipidemia is a major risk factor for CVD as the main cause of morbidity and mortality worldwide (18). Several studies have demonstrated the correlation of lipid profiles with CVD. However, the findings are controversial $(19,20)$. An important determinant of cardiovascular risk is AS, and few studies have investigated the association of lipids and related ratios with AS that measured by PWV (5), but not cAIx. In agreement with the current observation, Kawasoe et al. showed the association between TGs and PWV in a general Japanese population with normal low-density lipoprotein cholesterol (LDL-C) levels (21).

Moreover, observational studies found that TGs were positively associated with AS evaluated by PWV $(22,23)$. Conversely, Longitudinal study failed to reveal the significant association between TGs and AS measured by PWV. However, it has been shown that an increase in TGs levels of $48 \mathrm{mg} / \mathrm{dL}$ causes in a $1.0 \%$ higher PWV (24). Zhao et al. reported that the levels of TGs, total cholesterol, and non-HDL-C, except non-HDL-C/HDL-C ratio were not significantly associated with PWV in middle-aged and elderly Chinese participants (9). The discrepancies among studies attributed to differences in the characteristics of participants, age, and measurement methods of AS. The present study performed on the community-based sample with a healthy condition (self-declaration), unlike previous studies that selected participants with metabolic syndrome and diabetes $(22,24,25)$. Also, cardiovascular risk factors that can induce arterial wall damage and AS is higher in elderly subjects, which may impact on results of previous studies $(9,23)$.

Besides, we evaluated the systemic AS by cAIx that expresses the ratio of the augmented pressure to the pulse pressure, which provides information on the stiffness of the small vessels and large arteries (26). In agreement with the current analysis, Janner et al. performed the cross-sectional study for examining the association between AIx and cholesterol in different ages and both genders. The authors reported the association between AIx and cholesterol in both genders only in subjects aged less than 60 years (27). Another study did not identify a significant association of dyslipidemia with AS measured with AIx, whereas they reported a significant relationship between total cholesterol levels and AIx value (28).

Several potential mechanisms may explain the observed relationship between TGs, cholesterol, and AS. Elevated TGs may be induced reactive oxygen species and independently related to the insulin resistance and smaller LDL-C particles, which are more atherogenic (29). The effects of insulin resistance on AS can be mediated by the imbalance production of important AS' regulators, including nitric oxide and endothelin-1 (30). Insulin resistance may also lead to vessel wall hypertrophy and fibrosis, which can attribute to the activity of the renin-angiotensin-aldosterone system and expression of angiotensin II receptors in vascular tissue (30).

Anthropometric indices are associated with Arterial stiffness markers:

Several studies have also examined the association between conventional and novel anthropometric measures, and similar results have been obtained. In a study using healthy, hypertensive, and diabetic subjects, WHtR, ABSI, and BRI were found to be better predictors of PWV in both genders (31). Another study in obese and overweight people showed a relationship between PWV and visceral fat, BRI, and ABSI (32).

New anthropometric indices such as ABSI and BRI were developed due to the limitation of the traditional index (BMI) in the accurately reflect of BFM and visceral adipose tissue. The ABSI is independent of BMI, has been reported more closely associated with visceral adipose tissue, cardiovascular mortality, and risk of death than traditional anthropometric index such as BMI (33). In the present study, we found that ABSI was associated only with cAIx, but not PWV. Another parameter, BRI, based WC and height, has been suggested as a superior predictor of both BFM and visceral adiposity, compared to BMI (17). Some studies found that BRI had a potential predictive value to identify dyslipidemia and CVD $(34,35)$. However, a few studies have been conducted about BRI, and limited evidence has examined the association of BRI with AS indicators for predicting cardiovascular health status. The results of the present study demonstrated that BRI was associated with both PWV and cAIx, even after adjustment for potential confounders.

Common anthropometric indices, including BMI, WC, and WHR also assessed, and the results indicated that 
both WC and WHR were independently related to PWV and cAIx, but not BMI, which was consistent with other studies. BMI, as one of the most common anthropometric indices, cannot distinguish between adipose tissue and muscle mass, as well as peripheral and abdominal obesity (36). To eliminate this limitation, WC and WHR as substantial diagnostic components of metabolic syndrome and CVD are used alternatively as an indicator of abdominal obesity $(37,38)$. At the same time, WC and WHR could be used as an alternative to $\mathrm{BMI}$ and plays an essential role in assessing cardiovascular, metabolic risk (39). However, these parameters cannot distinguish between abdominal BFM and visceral fat. It should also be noted that height is not involved in calculating WC and WHR, which may lead to underestimated and overestimated in short and tall participants, respectively.

On the other hand, height can represent long-term nutrition and social status, which is likely to affect cardiometabolic risk (40), as Smith et al. indicated a negative association between height and CVD risk (41). To figure it out, WHtR was presented as a modification of WC with height, and we found that WHtR was related to cf-PWV. Some evidence showed that WHtR could assess cardiovascular risk factors and optimally used in predicting the incidence of metabolic syndrome in non-obese people, and is suggested as a screening tool for metabolic risk factors $(42,43)$. This means that WHtR is probably an appropriate index to evaluate abdominal obesity and can be used as a predictive value in metabolic syndrome, CVD, and AS.

In our analysis, we also included a visceral fat area and BFM, which independently associated with cAIx. Other studies using hypertensive, middle-aged, and older adults, reported that visceral fat was correlated with AS more closely than other parameters such as BMI and WC (44-46). This relationship may be explained by the positive association of visceral fat with free fatty acids and insulin levels that results in decreased nitric oxide production and increased sympathetic activity $(47,48)$. Indeed, we found that the $\mathrm{NC}$ of participants had a positive and negative correlation with PWV and cAIx, respectively. However, in the regression model, the relationship between this variable and AS was not significance, and it is not able to predict the AS value.

\section{Association of Physical Activity and arterial stiffness indices:}

In the current analysis, we also examined the relationship between AS and PA as a practical approach to preventing CVD, which is correlated with lower cardiovascular risk and mortality $(49,50)$. Our findings showed that PA was inversely related to cAIx, but not PWV, while this correlation is not clinically relevant. In line with our results, cross-sectional studies reporting the association of PA with cAIx and PWV; the authors reported an inverse relationship between PA and cAIx in hypertension and general participants below age 60 years $(27,51)$. A randomized control trial by Suboc et al. has shown that PA for twelve weeks had no significant effect on AS measured by PWV (52). However, another investigation displayed a negative association between low-PA and PWV (53); while Gomez-Marcos et al. found a positive relationship between low-PA and PWV (54). Several reasons can explain this discrepancy in the results; the methods used to evaluate PA (objective measures, different types of questionnaires), the characteristics of the sample, such as age and sex, the intensity of PA, and the season of the year.

To the best of our knowledge, this is the first study to assess the association of both PWV and cAIx with multiple body composition parameters (novel and traditional), bioelectrical impedance-derived parameters, lipid profiles, and PA. However, several limitations should be considered in the interpretation of our results. The associations which were assessed in the present study were based on cross-sectional designs, and therefore the cause-effect relationship is not clear. Finally, our study performed only on the Iranian population, which may be limited to the applicability of the results to other regions and ethnic groups. AS is likely to be influenced by Ethnicity and heredity (55), and anthropometric indices have various power to predict cardiovascular risk in different populations.

\section{Conclusion:}

In conclusion, our results showed that PWV as a local marker of AS was significantly associated with anthropometric indices including WC, WHR, WHtR, and BRI as well as TGs among lipid profiles, regardless of potential confounders. However, cAIx as a systemic marker of AS was independently associated with WC, 
WHR, visceral fat area and BFM, and novel anthropometric indices, namely ABSI and BRI, as well as cholesterol levels among lipid profiles.

Ethics approval and consent to participate: The Ethics Committee of the Mashhad University of Medical Sciences approved this study (Code of Ethics: IR.MUMS.MEDICAL.REC.1397.322). All participants signed a consent form approved by the ethics committee.

Consent for publication: Not Applicable.

Availability of data and materials: The data that support the findings of this study are available from [Dr. Saeed Eslami Hasan Abadi] but restrictions apply to the availability of these data, which were used under license for the current study, and so are not publicly available. Data are however available from the authors upon reasonable request and with permission of [Dr. Saeed Eslami Hasan Abadi].

Competing interests: The author has no relevant interests to declare.

Funding: This research did not receive any specific grant from funding agencies in the public, commercial, or not-for-profit sectors.

Authors' contributions: MN and SA drafted the manuscript. SEHA performed statistical analyses. HK, RR, MRSh, NSA made data ready and AN, SEHA, MN and SA interpreted the findings. RR, AN, MA made critical revision of the manuscript for key intellectual content. RR also takes full responsibility for data and the content of the manuscript. All authors read and approved the final manuscript.

Acknowledgments: This study was supported by the Iran Ministry of Health and Medical Education and Mashhad University of Medical Sciences as part of the "Persian cohort" research project.

\section{References:}

1. Cunha PG, Cotter J, Oliveira P, Vila I, Boutouyrie P, Laurent S, et al. Pulse wave velocity distribution in a cohort study: from arterial stiffness to early vascular aging. Journal of hypertension. 2015;33(7):1438-45.

2. Townsend RR, Wilkinson IB, Schiffrin EL, Avolio AP, Chirinos JA, Cockcroft JR, et al. Recommendations for Improving and Standardizing Vascular Research on Arterial Stiffness: A Scientific Statement From the American Heart Association. Hypertension. 2015;66(3):698-722.

3. Duprez DA, Cohn JN. Arterial stiffness as a risk factor for coronary atherosclerosis. Current atherosclerosis reports. 2007;9(2):139-44.

4. Zieman SJ, Melenovsky V, Kass DA. Mechanisms, pathophysiology, and therapy of arterial stiffness. Arteriosclerosis, Thrombosis, and Vascular Biology. 2005;25(5):932-43.

5. Cohn JN. Arterial stiffness, vascular disease, and risk of cardiovascular events. Circulation. 2006;113(5):601-3.

6. Laurent S, Boutouyrie P, Asmar R, Gautier I, Laloux B, Guize L, et al. Aortic stiffness is an independent predictor of all-cause and cardiovascular mortality in hypertensive patients. Hypertension (Dallas, Tex : 1979). 2001;37(5):1236-41.

7. Bolton E, Rajkumar C. The ageing cardiovascular system. Reviews in Clinical Gerontology. 2011;21(2):99109.

8. Desamericq G, Tissot CM, Akakpo S, Tropeano AI, Millasseau S, Macquin-Mavier I. Carotid-femoral pulse wave velocity is not increased in obesity. American journal of hypertension. 2015;28(4):546-51.

9. Zhao W, Gong W, Wu N, Li Y, Ye K, Lu B, et al. Association of lipid profiles and the ratios with arterial stiffness in middle-aged and elderly Chinese. Lipids in health and disease. 2014;13:37.

10. Cavalcante SL, Bohn L, Ribeiro F, Oliveira J. Physical Activity is Inversely Associated With Arterial Stiffness in Adult Males: A Brief Communication. Heart, Lung and Circulation. 2019;28(4):e29-e32. 
11. Andersson C, Lyass A, Larson MG, Spartano NL, Vita JA, Benjamin EJ, et al. Physical activity measured by accelerometry and its associations with cardiac structure and vascular function in young and middle-aged adults. Journal of the American Heart Association. 2015;4(3):e001528.

12. Rodrigues SL, Baldo MP, Lani L, Nogueira L, Mill JG, Sa Cunha R. Body mass index is not independently associated with increased aortic stiffness in a Brazilian population. American journal of hypertension. 2012;25(10):1064-9.

13. Poustchi H, Eghtesad S, Kamangar F, Etemadi A, Keshtkar AA, Hekmatdoost A, et al. Prospective Epidemiological Research Studies in Iran (the PERSIAN Cohort Study): Rationale, Objectives, and Design. Am J Epidemiol. 2018;187(4):647-55.

14. Goldberg G, Black A, Jebb S, Cole T, Murgatroyd P, Coward W, et al. Critical evaluation of energy intake data using fundamental principles of energy physiology: 1. Derivation of cut-off limits to identify under-recording. European journal of clinical nutrition. 1991;45(12):569-81.

15. Black A, Coward W, Cole T, Prentice A. Human energy expenditure in affluent societies: an analysis of 574 doubly-labelled water measurements. European journal of clinical nutrition. 1996;50(2):72-92.

16. Krakauer NY, Krakauer JC. A new body shape index predicts mortality hazard independently of body mass index. PloS one. 2012;7(7):e39504.

17. Thomas DM, Bredlau C, Bosy-Westphal A, Mueller M, Shen W, Gallagher D, et al. Relationships between body roundness with body fat and visceral adipose tissue emerging from a new geometrical model. Obesity (Silver Spring, Md). 2013;21(11):2264-71.

18. Go AS, Mozaffarian D, Roger VL, Benjamin EJ, Berry JD, Borden WB, et al. Heart disease and stroke statistics-2013 update: a report from the American Heart Association. Circulation. 2013;127(1):e6-e245.

19. Criqui MH, Heiss G, Cohn R, Cowan LD, Suchindran CM, Bangdiwala S, et al. Plasma triglyceride level and mortality from coronary heart disease. The New England journal of medicine. 1993;328(17):1220-5.

20. Di Angelantonio E, Sarwar N, Perry P, Kaptoge S, Ray KK, Thompson A, et al. Major lipids, apolipoproteins, and risk of vascular disease. Jama. 2009;302(18):1993-2000.

21. Kawasoe S, Ide K, Usui T, Kubozono T, Yoshifuku S, Miyahara H, et al. Association of Serum Triglycerides With Arterial Stiffness in Subjects With Low Levels of Low-Density Lipoprotein Cholesterol. Circulation journal : official journal of the Japanese Circulation Society. 2018;82(12):3052-7.

22. Kim HL, Lee JM, Seo JB, Chung WY, Kim SH, Zo JH, et al. The effects of metabolic syndrome and its components on arterial stiffness in relation to gender. Journal of cardiology. 2015;65(3):243-9.

23. Wang X, Ye P, Cao R, Yang X, Xiao W, Zhang Y, et al. Triglycerides are a predictive factor for arterial stiffness: a community-based 4.8-year prospective study. Lipids in health and disease. 2016;15:97.

24. Dabelea D, Talton JW, D'Agostino R, Jr., Wadwa RP, Urbina EM, Dolan LM, et al. Cardiovascular risk factors are associated with increased arterial stiffness in youth with type 1 diabetes: the SEARCH CVD study. Diabetes care. 2013;36(12):3938-43.

25. Kärkkäinen H, Saarelainen H, Valtonen P, Laitinen T, Raitakari OT, Juonala M, et al. Carotid artery elasticity decreases during pregnancy - the Cardiovascular Risk in Young Finns study. BMC Pregnancy Childbirth. 2014;14:98-.

26. Wilkinson IB, Prasad K, Hall IR, Thomas A, MacCallum H, Webb DJ, et al. Increased central pulse pressure and augmentation index in subjects with hypercholesterolemia. Journal of the American College of Cardiology. 2002;39(6):1005-11.

27. Janner JH, Godtfredsen NS, Ladelund S, Vestbo J, Prescott E. The association between aortic augmentation index and cardiovascular risk factors in a large unselected population. Journal of human hypertension. 
2012;26(8):476-84.

28. Razman M, Jamaluddin A, Ellyda M, Seikh F. Arterial Stiffness and its Association with Dyslipidemia. International Medical Journal Malaysia. 2013;12(2).

29. Lamarche B, Tchernof A, Moorjani S, Cantin B, Dagenais GR, Lupien PJ, et al. Small, dense low-density lipoprotein particles as a predictor of the risk of ischemic heart disease in men. Prospective results from the Quebec Cardiovascular Study. Circulation. 1997;95(1):69-75.

30. Jia G, Aroor AR, DeMarco VG, Martinez-Lemus LA, Meininger GA, Sowers JR. Vascular stiffness in insulin resistance and obesity. Front Physiol. 2015;6:231-.

31. Zhang J, Fang L, Qiu L, Huang L, Zhu W, Yu Y. Comparison of the ability to identify arterial stiffness between two new anthropometric indices and classical obesity indices in Chinese adults. Atherosclerosis. 2017;263:263-71.

32. Li G, Yao T, Wu XW, Cao Z, Tu YC, Ma Y, et al. Novel and traditional anthropometric indices for identifying arterial stiffness in overweight and obese adults. Clinical nutrition (Edinburgh, Scotland). 2020;39(3):893-900.

33. Dhana K, Kavousi M, Ikram MA, Tiemeier HW, Hofman A, Franco OH. Body shape index in comparison with other anthropometric measures in prediction of total and cause-specific mortality. Journal of epidemiology and community health. 2016;70(1):90-6.

34. Zhang K, Zhao Q, Li Y, Zhen Q, Yu Y, Tao Y, et al. Feasibility of anthropometric indices to identify dyslipidemia among adults in Jilin Province: a cross-sectional study. Lipids in health and disease. 2018;17(1):16.

35. Maessen MF, Eijsvogels TM, Verheggen RJ, Hopman MT, Verbeek AL, de Vegt F. Entering a new era of body indices: the feasibility of a body shape index and body roundness index to identify cardiovascular health status. PloS one. 2014;9(9):e107212.

36. Phillips CM, Tierney AC, Perez-Martinez P, Defoort C, Blaak EE, Gjelstad IM, et al. Obesity and body fat classification in the metabolic syndrome: impact on cardiometabolic risk metabotype. Obesity (Silver Spring, Md). 2013;21(1):E154-61.

37. Balkau B, Deanfield JE, Despres JP, Bassand JP, Fox KA, Smith SC, Jr., et al. International Day for the Evaluation of Abdominal Obesity (IDEA): a study of waist circumference, cardiovascular disease, and diabetes mellitus in 168,000 primary care patients in 63 countries. Circulation. 2007;116(17):1942-51.

38. Sumner AE, Sen S, Ricks M, Frempong BA, Sebring NG, Kushner H. Determining the waist circumference in african americans which best predicts insulin resistance. Obesity (Silver Spring, Md). 2008;16(4):841-6.

39. Ho SC, Chen YM, Woo JL, Leung SS, Lam TH, Janus ED. Association between simple anthropometric indices and cardiovascular risk factors. International journal of obesity and related metabolic disorders : journal of the International Association for the Study of Obesity. 2001;25(11):1689-97.

40. Gunnell D. Commentary: Early insights into height, leg length, proportionate growth and health. International journal of epidemiology. 2001;30(2):221-2.

41. Smith LG, Yatsuya H, Psaty BM, Longstreth WT, Jr., Folsom AR. Height and risk of incident intraparenchymal hemorrhage: Atherosclerosis Risk in Communities and Cardiovascular Health study cohorts. Journal of stroke and cerebrovascular diseases : the official journal of National Stroke Association. 2013;22(4):323-8.

42. Ashwell M, Gunn P, Gibson S. Waist-to-height ratio is a better screening tool than waist circumference and BMI for adult cardiometabolic risk factors: systematic review and meta-analysis. Obesity reviews : an official journal of the International Association for the Study of Obesity. 2012;13(3):275-86.

43. Zhu Q, Shen F, Ye T, Zhou Q, Deng H, Gu X. Waist-to-height ratio is an appropriate index for identifying cardiometabolic risk in Chinese individuals with normal body mass index and waist circumference. Journal 
of diabetes. 2014;6(6):527-34.

44. Natale F, Tedesco MA, Mocerino R, de Simone V, Di Marco GM, Aronne L, et al. Visceral adiposity and arterial stiffness: echocardiographic epicardial fat thickness reflects, better than waist circumference, carotid arterial stiffness in a large population of hypertensives. European journal of echocardiography : the journal of the Working Group on Echocardiography of the European Society of Cardiology. 2009;10(4):549-55.

45. Strasser B, Arvandi M, Pasha EP, Haley AP, Stanforth P, Tanaka H. Abdominal obesity is associated with arterial stiffness in middle-aged adults. Nutrition, metabolism, and cardiovascular diseases : NMCD. 2015;25(5):495-502.

46. Sutton-Tyrrell K, Newman A, Simonsick EM, Havlik R, Pahor M, Lakatta E, et al. Aortic stiffness is associated with visceral adiposity in older adults enrolled in the study of health, aging, and body composition. Hypertension (Dallas, Tex : 1979). 2001;38(3):429-33.

47. Wannamethee SG, Shaper AG, Durrington PN, Perry IJ. Hypertension, serum insulin, obesity and the metabolic syndrome. Journal of human hypertension. 1998;12(11):735-41.

48. Fantuzzi G, Mazzone T. Adipose tissue and atherosclerosis: exploring the connection. Arterioscler Thromb Vasc Biol. 2007;27(5):996-1003.

49. Myers J. Exercise and cardiovascular health. Circulation. 2003;107(1):e2-e5.

50. Kokkinos P. Physical activity, health benefits, and mortality risk. ISRN cardiology. 2012;2012.

51. O’Donovan C, Lithander FE, Raftery T, Gormley J, Mahmud A, Hussey J. Inverse relationship between physical activity and arterial stiffness in adults with hypertension. Journal of physical activity and health. 2014;11(2):272-7.

52. Suboc TB, Strath SJ, Dharmashankar K, Coulliard A, Miller N, Wang J, et al. Relative importance of step count, intensity, and duration on physical activity's impact on vascular structure and function in previously sedentary older adults. Journal of the American Heart Association. 2014;3(1):e000702.

53. Parsons TJ, Sartini C, Ellins EA, Halcox JPJ, Smith KE, Ash S, et al. Objectively measured physical activity, sedentary time and subclinical vascular disease: Cross-sectional study in older British men. Prev Med. 2016;89:194-9.

54. Gomez-Marcos MA, Recio-Rodriguez JI, Patino-Alonso MC, Agudo-Conde C, Lasaosa-Medina L, Rodriguez-Sanchez E, et al. Relationship between objectively measured physical activity and vascular structure and function in adults. Atherosclerosis. 2014;234(2):366-72.

55. Mitchell GF, DeStefano AL, Larson MG, Benjamin EJ, Chen MH, Vasan RS, et al. Heritability and a genome-wide linkage scan for arterial stiffness, wave reflection, and mean arterial pressure: the Framingham Heart Study. Circulation. 2005;112(2):194-9.

Table 1. Baseline characteristics of participants according to the tertiles of arterial stiffness markers by pulse wave velocity $(\mathrm{m} / \mathrm{s})$

\begin{tabular}{llll}
\hline & Tertiles of arterial stiffness markers & Tertiles of arterial stiffness markers & Tertiles of arter \\
\hline & T1 $\mathbf{( 5 . 9 8} \pm \mathbf{0 . 5 6})$ & $\mathbf{T 2} \mathbf{( 7 . 1 2} \pm \mathbf{0 . 2 6})$ & $\mathbf{T 3}(\mathbf{8 . 5 4} \pm \mathbf{0 . 8}$ \\
pulse wave velocity $(\mathbf{m} / \mathbf{s})$ & & & $43.90 \pm 7.4$ \\
Age (years) & $40.50 \pm 5.6$ & $41.00 \pm 5.8$ & 39.40 \\
Female (\%) & 67.60 & 46.50 & $1921 \pm 259$ \\
Energy intake (Kcal) & $1887 \pm 245$ & $1948 \pm 267$ & $25.80 \pm 2.4$ \\
BMI $\left(\mathrm{Kg} / \mathrm{m}^{2}\right)$ & $25.00 \pm 2.5$ & $25.10 \pm 2.4$ & $91.80 \pm 9.4$ \\
WC $(\mathrm{cm})$ & $87.40 \pm 7.3$ & $89.60 \pm 7.6$ & $0.92 \pm 0.06$ \\
WHR & $0.90 \pm 0.04$ & $0.91 \pm 0.04$ &
\end{tabular}




\begin{tabular}{llll}
\hline & Tertiles of arterial stiffness markers & Tertiles of arterial stiffness markers & Tertiles of arter \\
\hline WHtR & $0.53 \pm 0.04$ & $0.53 \pm 0.04$ & $0.54 \pm 0.05$ \\
ABSI & $0.07 \pm 0.00$ & $0.08 \pm 0.00$ & $0.08 \pm 0.00$ \\
BRI & $4.06 \pm 0.84$ & $4.06 \pm 0.88$ & $4.29 \pm 0.93$ \\
NC (cm) & $35.10 \pm 2.5$ & $36.10 \pm 2.3$ & $36.80 \pm 2.7$ \\
Visceral fat Area $\left(\mathrm{cm}^{2}\right)$ & $105.40 \pm 32.9$ & $102.40 \pm 35.6$ & $107.10 \pm 38.4$ \\
BFM (kg) & $21.90 \pm 5.4$ & $21.60 \pm 5.8$ & $22.70 \pm 6.5$ \\
FFM (kg) & $45.30 \pm 9.6$ & $49.00 \pm 9.8$ & $50.70 \pm 6.1$ \\
SBP (mmHg) & $97.70 \pm 12.9$ & $101.70 \pm 11.4$ & $104.50 \pm 13.4$ \\
DBP (mmHg) & $65.10 \pm 8.2$ & $67.60 \pm 7.6$ & $69.80 \pm 9.1$ \\
HR (bpm) & $66.90 \pm 8.0$ & $88.80 \pm 9.3$ & $68.50 \pm 10.3$ \\
FBS (mg/dL) & $88.50 \pm 11.1$ & $97.50(65.0-134.0)$ & $90.20 \pm 14.7$ \\
TG (mg/dL) & $81.00(59.5-119.5)$ & $182.60 \pm 37.3$ & $105.00(75.0-15$ \\
TC (mg/dL) & $175.20 \pm 36.6$ & $58.50 \pm 14.8$ & $185.30 \pm 36.4$ \\
HDL-C (mg/dL) & $59.90 \pm 14.0$ & $124.00 \pm 36.4$ & $57.80 \pm 13.2$ \\
Non-HDL-C & $115.30 \pm 36.2$ & $2.20 \pm 0.9$ & $127.50 \pm 36.3$ \\
Non-HDL-C/HDL-C & $2.00 \pm 0.8$ & $38.90 \pm 4.2$ & $2.30 \pm 0.9$ \\
Total MET(MET-hour/day) & $38.60 \pm 3.7$ & & $38.10 \pm 4.1$ \\
\hline
\end{tabular}

BMI, body mass index; WC, waist circumference; WHR, waist to hip ratio; WHtR, waist-to-height ratio; ABSI, a body shape index; BRI, body roundness index; NC, neck circumferences; BFM, body fat mass; FFM, fat free mass; SBP, systolic blood pressure; DBP, diastolic blood pressure; HR, heart rate; FPG, fasting plasma glucose; TGs, triglycerides; HDL-C, high-density lipoprotein cholesterol; TC, total cholesterol; MET, metabolic equivalent of task.

Data represented as mean \pm SD or median (25th-75th percentile) for continuous variables (except age) or percent for categorical variables

Linear regression and $\chi^{2}$ were used to test the trend of continuous and categorical variables, respectively, according to tertiles.

Table 2. Baseline characteristics of participants according to the tertiles of arterial stiffness markers by central augmentation index $(\%)$

\begin{tabular}{lllll}
\hline & T1 $(\mathbf{5 . 9 8} \pm \mathbf{0 . 5 6})$ & $\mathbf{T 2}(\mathbf{7 . 1 2} \pm \mathbf{0 . 2 6})$ & $\mathbf{T 3} \mathbf{( 8 . 5 4} \pm \mathbf{0 . 8 5})$ & $\mathbf{P}$ for trend \\
\hline central augmentation index (\%) & & & & \\
Age (years) & $39.60 \pm 4.9$ & $41.60 \pm 6.5$ & $44.30 \pm 7.0$ & $\mathbf{i} \mathbf{0 . 0 0 1}$ \\
Female (\%) & 35.9 & 58.0 & 59.9 & $\mathbf{i} \mathbf{0 . 0 0 1}$ \\
Energy intake (Kcal) & $2056 \pm 260$ & $1901 \pm 217$ & $1796 \pm 228$ & $\mathbf{i} \mathbf{0 . 0 0 1}$ \\
BMI (Kg/m $\left.{ }^{2}\right)$ & $24.90 \pm 2.4$ & $25.50 \pm 2.4$ & $25.40 \pm 2.6$ & 0.053 \\
WC (cm) & $89.00 \pm 7.9$ & $89.90 \pm 7.8$ & $89.80 \pm 9.4$ & 0.346 \\
WHR & $0.90 \pm 0.05$ & $0.91 \pm 0.04$ & $0.91 \pm 0.05$ & 0.072 \\
WHtR & $0.52 \pm 0.04$ & $0.54 \pm 0.04$ & $0.54 \pm 0.05$ & $\mathbf{i} \mathbf{0 . 0 0 1}$ \\
ABSI & $0.08 \pm 0.00$ & $0.08 \pm 0.00$ & $0.08 \pm 0.00$ & 0.143 \\
BRI & $3.90 \pm 0.81$ & $4.23 \pm 0.86$ & $4.27 \pm 0.95$ & $\mathbf{i} \mathbf{0 . 0 0 1}$ \\
NC (cm) & $36.30 \pm 2.6$ & $35.90 \pm 2.4$ & $35.70 \pm 2.7$ & $\mathbf{0 . 0 1 9}$ \\
Visceral fat Area $\left(\mathrm{cm}^{2}\right)$ & $94.60 \pm 32.7$ & $109.30 \pm 34.9$ & $111.00 \pm 37.5$ & $\mathbf{i} \mathbf{0 . 0 0 1}$ \\
BFM (kg) & $20.60 \pm 5.8$ & $22.80 \pm 5.7$ & $22.90 \pm 6.0$ & $\mathbf{i} \mathbf{0 . 0 0 1}$ \\
FFM (kg) & $51.50 \pm 10.6$ & $47.40 \pm 9.5$ & $46.20 \pm 9.4$ & $\mathbf{i} \mathbf{0 . 0 0 1}$ \\
SBP (mmHg) & $102.10 \pm 12.3$ & $100.30 \pm 12.6$ & $101.70 \pm 13.8$ & 0.729 \\
DBP (mmHg) & $67.70 \pm 8.6$ & $67.40 \pm 8.1$ & $67.40 \pm 8.8$ & 0.716
\end{tabular}




\begin{tabular}{lllll}
\hline & T1 $\mathbf{( 5 . 9 8} \pm \mathbf{0 . 5 6})$ & $\mathbf{T 2}(\mathbf{7 . 1 2} \pm \mathbf{0 . 2 6})$ & $\mathbf{T 3}(\mathbf{8 . 5 4} \pm \mathbf{0 . 8 5})$ & $\mathbf{P}$ for trend \\
\hline HR $(\mathrm{bpm})$ & $66.80 \pm 9.1$ & $68.20 \pm 9.7$ & $68.20 \pm 8.4$ & 0.114 \\
FBS (mg/dL) & $89.70 \pm 12.1$ & $89.20 \pm 14.0$ & $88.30 \pm 11.7$ & 0.250 \\
TG (mg/dL) & $92.00(67.0-125.0)$ & $94.00(66.0-136.0)$ & $93.00(67.0-144.0)$ & 0.401 \\
TC (mg/dL) & $175.40 \pm 32.9$ & $180.60 \pm 38.2$ & $186.50 \pm 38.5$ & $\mathbf{0 . 0 0 2}$ \\
HDL-C (mg/dL) & $57.50 \pm 14.0$ & $58.10 \pm 13.3$ & $60.60 \pm 14.5$ & $\mathbf{0 . 0 1 9}$ \\
Non-HDL-C & $117.90 \pm 33.2$ & $122.40 \pm 38.0$ & $125.80 \pm 37.9$ & $\mathbf{0 . 0 2 4}$ \\
Non-HDL-C/HDL-C & $2.20 \pm 0.9$ & $2.20 \pm 0.9$ & $2.20 \pm 0.8$ & 0.975 \\
Total MET(MET-hour/day) & $39.00 \pm 4.6$ & $38.20 \pm 3.3$ & $38.40 \pm 4.0$ & 0.152 \\
\hline
\end{tabular}

BMI, body mass index; WC, waist circumference; WHR, waist to hip ratio; WHtR, waist-to-height ratio; ABSI, a body shape index; BRI, body roundness index; NC, neck circumferences; BFM, body fat mass; FFM, fat free mass; SBP, systolic blood pressure; DBP, diastolic blood pressure; HR, heart rate; FPG, fasting plasma glucose; TGs, triglycerides; HDL-C, high-density lipoprotein cholesterol; TC, total cholesterol; MET, metabolic equivalent of task.

Data represented as mean \pm SD or median (25th-75th percentile) for continuous variables (except age) or percent for categorical variables

Linear regression and $\chi^{2}$ were used to test the trend of continuous and categorical variables, respectively, according to tertiles.

Table 3. Correlation of arterial stiffness markers with anthropometric indices, lipid profiles, and PA.

\begin{tabular}{|c|c|c|c|c|}
\hline & $\begin{array}{l}\text { pulse wave } \\
\text { velocity }\end{array}$ & $\begin{array}{l}\text { pulse wave } \\
\text { velocity }\end{array}$ & $\begin{array}{l}\text { central } \\
\text { augmentation } \\
\text { index }\end{array}$ & $\begin{array}{l}\text { central } \\
\text { augmentation } \\
\text { index }\end{array}$ \\
\hline & correlation & $\mathrm{p}$ value & correlation & $\mathrm{p}$ value \\
\hline \multicolumn{5}{|l|}{ Anthropometry } \\
\hline $\mathrm{BMI}\left(\mathrm{Kg} / \mathrm{m}^{2}\right)$ & 0.135 & 0.001 & 0.062 & 0.118 \\
\hline $\mathrm{WC}(\mathrm{cm})$ & 0.208 & ¡ 0.001 & 0.002 & 0.957 \\
\hline WHR & 0.182 & i 0.001 & 0.049 & 0.207 \\
\hline WHtR & 0.129 & 0.001 & 0.159 & i 0.001 \\
\hline ABSI & 0.091 & 0.021 & 0.043 & 0.274 \\
\hline BRI & 0.112 & 0.005 & 0.163 & i 0.001 \\
\hline $\mathrm{NC}(\mathrm{cm})$ & 0.242 & i 0.001 & -0.131 & 0.001 \\
\hline $\begin{array}{l}\text { Visceral fat Area } \\
\left(\mathrm{cm}^{2}\right)\end{array}$ & 0.026 & 0.507 & 0.191 & ¡ 0.001 \\
\hline BFM (kg) & 0.057 & 0.144 & 0.149 & i 0.001 \\
\hline FFM (kg) & 0.198 & i 0.001 & 0.256 & ¡ 0.001 \\
\hline \multicolumn{5}{|l|}{ Lipid profiles } \\
\hline TG (mg/dL) & 0.187 & ¡ 0.001 & 0.060 & 0.126 \\
\hline TC (mg/dL) & 0.140 & ¡ 0.001 & 0.137 & i 0.001 \\
\hline HDL-C (mg/dL) & -0.036 & 0.360 & 0.102 & 0.009 \\
\hline Non-HDL & 0.155 & ¡ 0.001 & 0.099 & 0.011 \\
\hline Non-HDL/HDL & 0.123 & 0.002 & 0.003 & 0.934 \\
\hline \multicolumn{5}{|l|}{$\begin{array}{l}\text { Physical } \\
\text { activity }\end{array}$} \\
\hline PA groups & -0.051 & 0.197 & -0.067 & 0.086 \\
\hline
\end{tabular}


BMI, body mass index; WC, waist circumference; WHR, waist to hip ratio; WHtR, waist-to-height ratio; ABSI, a body shape index; BRI, body roundness index; NC, neck circumferences; BFM, body fat mass; FFM, fat-free mass; SBP, systolic blood pressure; DBP, diastolic blood pressure; TGs, triglycerides; HDL-C, high-density lipoprotein cholesterol; TC, total cholesterol; MET, metabolic equivalent of task.

Effect estimates with a p-value $<0.05$ are indicated in

bold.

Table 4. Association of arterial stiffness markers with anthropometric indices, lipid profiles, and PA.

\begin{tabular}{|c|c|c|c|c|}
\hline & pulse wave velocity & pulse wave velocity & pulse wave velocity & central augmentation ir \\
\hline & Unstandardized $\beta$ (95\% CI) & Standardized $\beta$ & $\mathrm{p}$ value & Unstandardized $\beta(95 \%$ \\
\hline \multicolumn{5}{|l|}{ anthropometry } \\
\hline $\mathrm{BMI}\left(\mathrm{Kg} / \mathrm{m}^{2}\right)$ & $0.029(-0.016$ to 0.075$)$ & 0.060 & 0.209 & $0.042(-0.336$ to 0.420$)$ \\
\hline $\mathrm{WC}(\mathrm{cm})$ & $0.016(0.002$ to 0.030$)$ & 0.110 & 0.023 & $0.125(0.012$ to 0.23 \\
\hline WHR & $2.060(0.131$ to 3.989$)$ & 0.088 & 0.036 & $18.153(2.260$ to 34.0 \\
\hline WHtR & $2.512(0.273$ to 4.752$)$ & 0.097 & 0.028 & 13.557 (-4.999 to 32.112 \\
\hline ABSI & $22.864(-6.930$ to 52.658$)$ & 0.061 & 0.132 & $298.587(54.543$ to 5 \\
\hline BRI & $0.083(0.003$ to 0.162$)$ & 0.088 & 0.041 & $0.076(0.000$ to 0.152 \\
\hline $\mathrm{NC}(\mathrm{cm})$ & $0.057(-0.008$ to 0.121$)$ & 0.124 & 0.084 & $0.262(-0.271$ to 0.794$)$ \\
\hline Visceral fat Area $\left(\mathrm{cm}^{2}\right)$ & $0.002(-0.001$ to 0.005$)$ & 0.067 & 0.151 & $0.028(0.002$ to 0.05 \\
\hline BFM (kg) & $0.016(-0.003$ to 0.034$)$ & 0.076 & 0.092 & $0.155(0.004$ to 0.307 \\
\hline FFM (kg) & $-0.103(-0.378$ to 0.172$)$ & -0.862 & 0.463 & $1.634(-0.621$ to 3.890$)$ \\
\hline \multicolumn{5}{|l|}{ Lipid profiles } \\
\hline $\mathrm{TG}(\mathrm{mg} / \mathrm{dL})$ & $0.199(0.039$ to 0.360$)$ & 0.106 & 0.015 & $0.904(-0.495$ to 2.304$)$ \\
\hline $\mathrm{TC}(\mathrm{mg} / \mathrm{dL})$ & $0.002(-0.001$ to 0.004$)$ & 0.057 & 0.165 & $0.023(0.001$ to 0.045 \\
\hline HDL-C (mg/dL) & $0.002(-0.005$ to 0.009$)$ & 0.021 & 0.611 & $0.025(-0.032$ to 0.082$)$ \\
\hline Non-HDL & $0.001(-0.001$ to 0.003$)$ & 0.025 & 0.549 & $0.012(-0.005$ to 0.030$)$ \\
\hline Non-HDL/HDL & $0.016(-0.065$ to 0.097$)$ & 0.016 & 0.703 & $0.305(-0.396$ to 1.005$)$ \\
\hline \multicolumn{5}{|l|}{ Physical activity } \\
\hline PA groups & $-0.015(-0.039$ to 0.008$)$ & -0.051 & 0.206 & $-0.213(-0.407$ to $-\mathrm{C}$ \\
\hline
\end{tabular}

BMI, body mass index; WC, waist circumference; WHR, waist to hip ratio; WHtR, waist-to-height ratio; ABSI, a body shape index; BRI, body roundness index; NC, neck circumferences; BFM, body fat mass; FFM, fat free mass; SBP, systolic blood pressure; DBP, diastolic blood pressure; TGs, triglycerides; HDL-C, high-density lipoprotein cholesterol; TC, total cholesterol; MET, metabolic equivalent of task.

adjusted for age, sex, SBP, DBP, HR, FBS, BUN, Cr, ALT, AST, ALP, skeletal muscle mass, sleep duration, total energy, alcohol consumption, and smoking

Effect estimates with a p-value $<0.05$ are indicated inbold .

Figure 1: Comparison of mean values of arterial stiffness markers among their tertiles, stratified by gender 

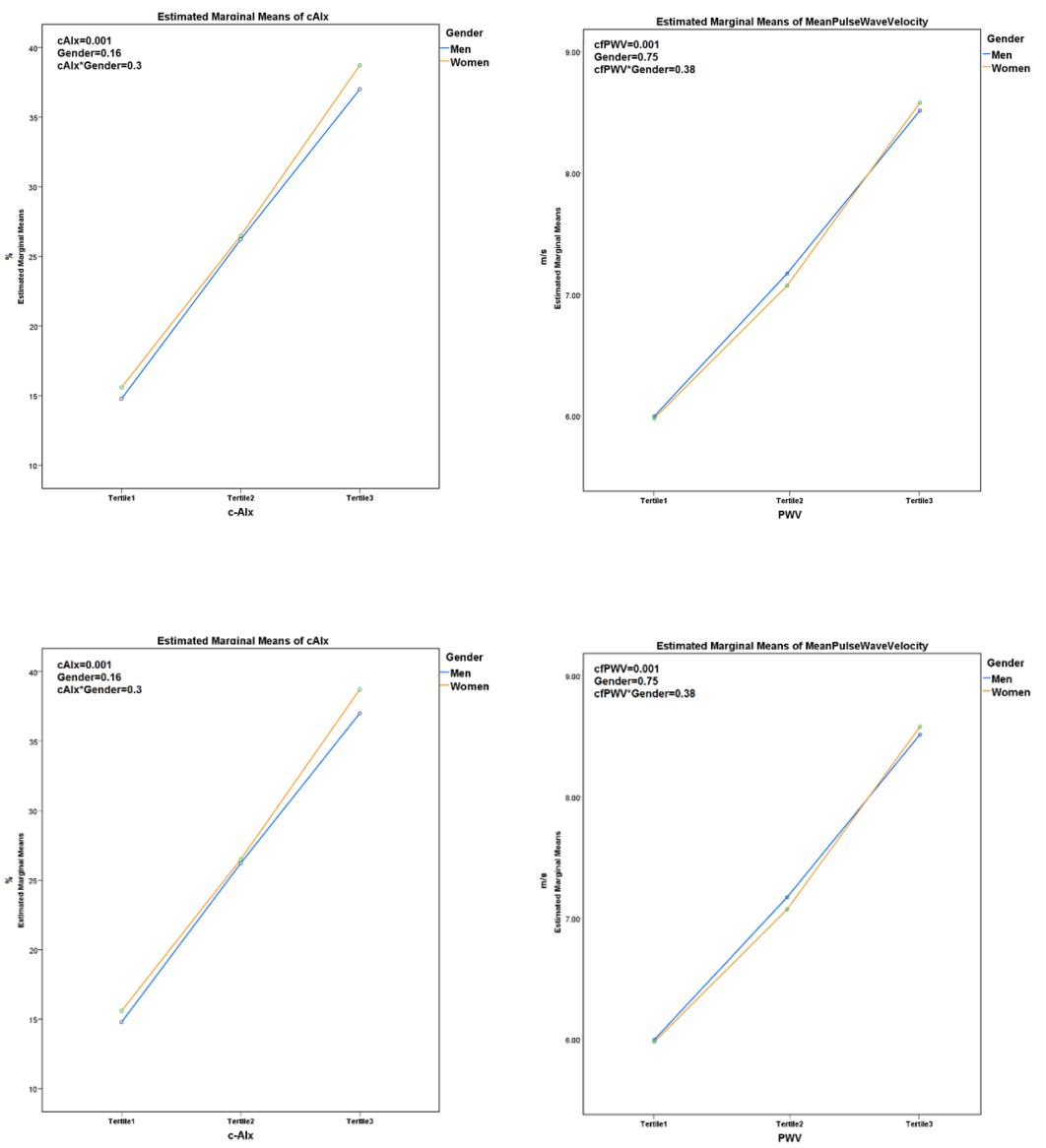